birth and risk of preterm birth, But there are few analyses in which outcomes of birth, within a specific country, are classified by both mother's country of birth and ethnicity.

Setting Live singleton births in England and Wales of babies whose ethnicity was recorded as being Black African or Black Caribbean in 2005 and 2006.

Aim To compare rates of preterm birth and low birth weight in this group of babies born to mothers born in African and Caribbean countries or England and Wales.

Method In England and Wales birth weight and mother's country of birth are recorded at birth registration whereas ethnic group of baby and gestational age are recorded in the data set generated when the NHS number, a national unique patient identifier, is issued. Linking these two data sets has made it possible to assess the association between mother's country of birth, baby's ethnicity and birth outcomes. Data from the linked data set were used for the analysis. Countries were grouped according to UN geographical regions.

Results Mothers of babies of African ethnicity, born in Eastern or Northern Africa had significantly lower odds than those born in England and Wales of having a preterm baby. This remained significant after adjusting for mother's age at birth and sex of baby. In terms of low birth weight, after adjusting for gender, mother's age at birth and gestational age, mothers of babies of African ethnicity born in Middle and Western Africa had significantly lower odds of having a low birth weight baby compared with those born in England and Wales. Similarly, after adjusting for the available confounders, mothers of babies of Caribbean ethnicity, born in the Caribbean countries had lower odds of having a low birth weight baby compared with mothers born in England and Wales.

Conclusion Generally, preterm birth and low birth weight rates of babies of African or Caribbean migrant women born in England and Wales seems to be higher than those who migrated to England and Wales having themselves been born in African or Caribbean countries. Further research is needed about the possible causes of this difference in birth outcomes.

\section{P48 MODIFYING HEALTH PROMOTION INTERVENTIONS FOR ETHNIC MINORITY GROUPS: SYSTEMATIC OVERVIEW OF GUIDELINES AND REVIEWS}

doi:10.1136/jech.2010.120477.48

${ }^{1} \mathrm{E}$ Davidson, ${ }^{1} \mathrm{~J} \mathrm{~J}$ Liu, ${ }^{2} \mathrm{U}$ Yousuf, ${ }^{3} \mathrm{R}$ Bhopal, ${ }^{4} \mathrm{M}$ Johnson, ${ }^{5} \mathrm{M}$ White, ${ }^{6} \mathrm{G}$ Netto, ${ }^{7} \mathrm{M}$ Deverill, ${ }^{1} \mathrm{~A}$ Sheikh. ${ }^{1}$ General Practice section, Centre for Population Health Sciences, The University of Edinburgh, Medical School, Edinburgh, UK; ${ }^{2}$ Aberdeen Royal Infirmary, Aberdeen, UK; ${ }^{3}$ Public Health Sciences section, Centre for Population Health Sciences, University of Edinburgh, Medical School, Edinburgh, UK; ${ }^{4}$ Mary Seacole Research Centre, De Montfort University, Leicester, UK; ${ }^{5}$ Institute of Health \& Society, Faculty of Medical Sciences, Newcastle University, Newcastle upon Tyne, UK; ${ }^{6}$ School of the Built Environment, Heriot-Watt University, Edinburgh, UK; ${ }^{7}$ Institute of Health and Society, University of Newcastle, Newcastle upon Tyne, UK

Background Some UK ethnic minority groups experience disproportionate levels of morbidity and mortality when compared with the majority White population. For these populations, access to and use of health promotion interventions may be limited. Adaptation of smoking cessation, physical activity and nutrition interventions of proven effectiveness for the majority population could represent an efficient strategy for reducing persistent health inequalities when adapted for minority ethnic populations.

Objectives To identify the high-level evidence for health promotion interventions which have proven effectiveness for the general population and construct a framework of effective interventions, including any recommendations relating to ethnic minority populations.

Design A systematic overview was conducted with two reviewers independently searching and identifying guidelines and systematic reviews of interventions for smoking cessation, improving nutrition and physical activity. SIGN, NICE and Clinical Evidence databases were searched for relevant guidelines. Cochrane Library, Campbell Collection, HTA reviews and DARE databases were searched for systematic reviews. Data on the effectiveness of interventions were extracted

Results 19 guidelines were identified as relevant. 2399 systematic review records were identified and assessed for eligibility. 187 systematic reviews were included in the final analysis. The guidelines revealed a large evidence base for smoking cessation interventions, but highlighted major gaps in relation to how best to increase physical activity and improve nutrition. There was little advice in these guidelines on how to adapt interventions to meet the needs of ethnic minority populations. The 187 systematic reviews were screened to identify any additional effective interventions not included in the guidelines. All effective, evidence-based interventions have been compiled into a summary framework. The 187 systematic reviews were also subjected to a detailed assessment of the population composition to determine whether any subgroup analysis for ethnic minority groups was undertaken. Approximately half of the reviews reported the inclusion of ethnic minority groups; however, no reviews conducted subgroup analyses according to ethnicity and ethnic-specific recommendations were scarce.

Conclusions The evidence base reviewed provides specific guidance on effective interventions for smoking cessation, but generic advice for increasing physical activity and improving nutrition. Identification of the range of evidence-based interventions for these three areas has led to the development of a summary framework that can be utilised for health promotion. Interventions already found to be effective in the majority population are, if appropriately adapted, likely to prove effective in minority ethnic populations. This work will advance current guidance on how to approach adaptation.

\section{P49 MODIFYING HEALTH PROMOTION INTERVENTIONS FOR ETHNIC MINORITY GROUPS: SYSTEMATIC REVIEW OF EMPIRICAL EVIDENCE}

doi:10.1136/jech.2010.120477.49

${ }^{1} \mathrm{~J} J$ Liu, ${ }^{1} E$ Davidson, ${ }^{2} \mathrm{R}$ Bhopal, ${ }^{3} \mathrm{M}$ Johnson, ${ }^{4} \mathrm{M}$ White, ${ }^{5} \mathrm{M}$ Deverill, ${ }^{6} \mathrm{G}$ Netto, ${ }^{1}$ A Sheikh. ${ }^{1}$ General Practice section, Centre for Population Health Sciences, The University of Edinburgh, Medical School, Edinburgh, UK; ${ }^{2}$ Public Health Sciences section, Centre for Population Health Sciences, University of Edinburgh, Medical School, Edinburgh, UK; ${ }^{3}$ Mary Seacole Research Centre, De Montfort University, Leicester, UK; ${ }^{4}$ Institute of Health \& Society, Faculty of Medical Sciences, Newcastle University, Newcastle upon Tyne, UK; ${ }^{5}$ Institute of Health and Society, University of Newcastle, Newcastle upon Tyne, UK; ${ }^{6}$ School of the Built Environment, Heriot-Watt University, Edinburgh, UK

Background Health promotion interventions have proved to be costeffective strategies to reduce morbidity and mortality associated with smoking, physical inactivity and poor diet in the general population. Some ethnic minority groups are disproportionately affected by these lifestyle factors, and existing evidence suggests that adapting evidence-based health promotion interventions for these populations may prove to be an effective strategy to tackle health inequalities.

Objectives To identify health promotion interventions for smoking cessation, increasing physical activity and improving nutrition which have been adapted for African-Caribbean, South Asian and Chinese-origin populations and to document how this has been achieved and with what effect.

Design A systematic review was conducted with two reviewers independently searching, identifying, extracting and critically appraising empirical studies of adapted interventions. The databases searched include MEDLINE, EMBASE, ASSIA, Psycinfo, 